\title{
A INFLUÊNCIA DO VÍDEO DE INFORMAÇÃO ADICIONAL EM PACIENTES SUBMETIDAS À MASTECTOMIA: O ESTUDO DA ANSIEDADE
}

\author{
Roberto Henrique Amorim de Medeiros* \\ Maria Lúcia Tiellet Nunes
}

\begin{abstract}
RESUMO. A ansiedade é um sintoma de alta incidência nos pacientes durante o tratamento dos casos de câncer. No câncer de mama, os quadros ansiosos podem aparecer em consequiência do próprio diagnóstico ou imediatamente após à cirurgia mutiladora. Este trabalho traz os primeiros resultados de uma pesquisa em andamento, elaborada para investigar os possíveis efeitos de um procedimento moderno de informação na avaliação da percepção de dor de pacientes submetidas à mastectomia no Serviço de Mastologia do Hospital de Clínicas de Porto Alegre. Uma amostra de 22 pacientes foi estudada segundo o delineamento experimental clássico, com dois grupos. $\mathrm{O}$ vídeo de informação foi apresentado no período pré-operatório apenas ao grupo experimental. Ambos os grupos foram avaliados em termos de traço e estado de ansiedade, através do Inventário de Ansiedade Traço e Estado (IDATE). Os resultados revelaram aumento dos índices de Estado de Ansiedade no grupo controle na comparação pré e pós-operatória. Esses achados parecem indicar a utilidade do vídeo de informação com vistas ao bem-estar das pacientes. Sugere-se, entretanto, o aumento da amostra da pesquisa para a melhor avaliação das tendências encontradas.
\end{abstract}

Palavras-chaves: video, ansiedade, mastectomia.

\section{THE INFLUENCE OF ADDITIONAL INFORMATION VIDEO IN PATIENTS SUBMITTED TO MASTECTOMY: AN ANXIETY STUDY}

\begin{abstract}
Anxiety is a high incidence symptom in the treatment of cancer patients. In the case of breast cancer, anxiety can occur after the diagnosis or shortly after a mutilating surgery. This paper presents the preliminary results of an ongoing research aiming to investigate the effects of modern means of information in the evaluation of pain perception in mastectomy patients in the Mastectomy Centre of the "Hospital das Clínicas" in Porto Alegre, South of Brazil. A sample of 22 patients divided into two groups was studied using a standard experimental method. An informational video was shown to one of the groups in the pre-operation period. The anxiety of both groups was evaluated by the Portuguese version of the State-Trait Anxiety Inventory. The results showed higher scores of anxiety in the control group after surgery. Such data reveal the benefits of using video information to increase the well being of these patients. However, a larger sample is recommended in order to enhance the results.
\end{abstract}

Key words: video, anxiety, mastectomy.

\section{INTRODUÇÃO}

O número de casos de câncer de mama catalogados nos estádios iniciais da doença é muito reduzido, tornando a mastectomia com linfadenectomia axilar uma intervenção freqüente.
Quando a terapêutica escolhida constituir-se num procedimento invasivo, como é o caso da cirurgia, os níveis de angústia dos pacientes tendem a aumentar consideravelmente, trazendo consigo efeitos indesejáveis ao tratamento (Horne, Vatmanidis e Careri, 1994; Clewes e Endler, 1994; Nuñez,

Psicólogo, especialista em atendimento clínico individual com ênfase em psicanálise, Clínica de Atendimento Psicológico da UFRGS, Mestre em Psicologia Clínica, PPG Psicologia, Pontíficia Universidade Católica do Rio Grande do Sul-PUCRS.

Endereço para correspondência: R. Eng ${ }^{\circ}$. Vespúcio de Abreu, 36/32, Santana, Porto Alegre-RS. E-mail: robertoamorim80@hotmail.com

\# Psicóloga, doutora em Psicologia Clínica (Universidade Livre de Berlim, 1989), professora dos cursos de graduação e pósgraduação da Faculdade de Psicologia da Pontíficia Universidade Católica do Rio Grande do Sul-PUCRS. 
Sanabria, Guerrero e Sanchez, 1991; Heywood, Blackie, Cameron e Dowell, 1998).

A ciência psicológica nos ensina que o atendimento às demandas psíquicas dos pacientes traz incrementos ao bem-estar, fazendo com que eles se percebam melhor, participem de modo efetivo na sua melhora, otimizam o uso de seu potencial, colaborando, assim, com os profissionais que os atendem (Campos, 1995).

O bem-estar pode ser compreendido como o sentimento proveniente de um certo número de fatores geradores de prazer para o indivíduo como resultado da combinação de aspectos demográficos, biológicos e de cunho social. Os fatores podem ser tanto de ordem somática como psíquica e, em alguns casos, até mesmo possuir um caráter psicossomático. Incluem-se entre relações interpessoais satisfatórias: processos familiares normais, lazer, condições de vida e de trabalho adequadas, independência e autonomia, ausência de conseqüências somáticas e psíquicas de enfermidade, ausência de problemas econômicos, participação em atividades comunitárias, saúde subjetiva e objetiva, satisfação com a vida, a família, os amigos e vizinhos, boa moradia, entre outros fatores. (Fernández-Ríos, 1994).

Segundo os modernos estudos na área, a ansiedade constitui um dos fatores mais influentes no sentimento de bem-estar dos indivíduos. É recomendável, portanto, a verificação desse aspectos para uma melhor compreensão, diagnóstico e tratamento da questão do bem-estar em pacientes cirúrgicos (Payne, Hoffman, Theodoulou, Dosik e Masie, 1999; Thomas, Robinson, Champion, McKell, Pell, 1998; Walls, Lord e Bogduk, 1997; Page, Verhoef e Emes, 1995; Kuecuekdeveci, Tennant, Hardo e Chamberlain, 1996; Becker e cols., 1997; Farragher, 1997; Morasso, 1997; O’Mahoney e Carrol, 1997; Tasmuth, Estlanderb e Kalso, 1996).

A mastectomia é, sem dúvida, o procedimento mais traumático da terapêutica do câncer de mama. A ansiedade é um sintoma de incidência bastante comum nas pacientes após o diagnóstico, durante o tratamento e principalmente naquelas que precisam se submeter à cirurgia (Ferrel, 1996). Acredita-se que o estudo da ansiedade em pacientes mastectomizadas é importante para a identificação daquelas pacientes com maior risco de desenvolver distúrbios psicopatológicos no decorrer do tratamento. (Payne e cols., 1999; Morasso, 1997)

O câncer de mama demanda um tempo prolongado de tratamento, exames diagnósticos e de controle, até se chegar à cirurgia. Horne, Vatmanidis e Careri (1994) indicam que pacientes submetidos a um procedimento médico invasivo requerem uma maior atenção quanto ao que acreditam estar ocorrendo, quanto ao que pensam sobre a necessidade da operação e seus conhecimentos sobre a mesma, bem como quanto às suas habilidades em decodificar para si próprios as informações que recebem.

Breemhaar, Van den Borne e Mullen (1996), num estudo em que acompanhava pacientes desde a admissão no hospital até a sua saída, constataram neles a presença de problemas emocionais, devido ao medo da cirurgia e da anestesia, bem como pela falta de informação sobre detalhes médicos, funções dos profissionais do hospital e a respeito do final do tratamento. Conclusões semelhantes foram produzidas através de uma análise da comunicação entre cirurgiões e seus pacientes durante consultas de rotina (Levinson e Chaumeton, 1999).

Glanz e Lerman (1992) afirmaram que mais de $25 \%$ das mulheres passam a sofrer alguma morbidade psicológica, como quadros ansiosos, após o diagnóstico de câncer no seio ou durante o tratamento específico. Experimentos atuais têm encontrado índices ainda maiores desse fenômeno, chegando a cerca de $60 \%$ dos casos (Brum, Chem, Peres e Magalhães, 1997). A melhora de pacientes que recebem uma maior atenção durante o tratamento hospitalar ocorre durante o pós-operatório e também se faz presente na diminuição da recorrência da doença ou da necessidade de novos cuidados (Appels, Bar, Lasker e Flamm, 1997).

Para o ano de 2001, o Instituto Nacional do Câncer (INCA) estima o surgimento de 31.590 novos casos de câncer de mama no país. A estimativa para óbitos pode chegar ao número de 8.670 a cada 100.000 mulheres. No ano 2000 os estados com mais afetados pela doença no Brasil são Rio Grande do Sul, São Paulo, Rio de Janeiro, com níveis de incidência estimados entre $44,7 \%$ a $72,8 \%$ a cada 100.000 mulheres estudadas.

Em face das condições alarmantes de incidência, sobrevivência e mortalidade subjacentes ao câncer de mama, o presente trabalho foi elaborado com o intuito de investigar os possíveis efeitos de um procedimento de informação, na avaliação da percepção de dor de pacientes submetidas à mastectomia no Serviço de Mastologia do Hospital de Clínicas de Porto Alegre (HCPA). O procedimento foi realizado através da apresentação de um vídeo informativo previamente à cirurgia. Testes administrados antes e depois do ato cirúrgico garantiram a verificação e o estudo de um dos principais aspectos influentes no sentimento de bem-estar, ou seja, a ansiedade. Este trabalho traz os primeiros resultados de uma pesquisa em andamento 
que procurará verificar o tipo de influência que o procedimento de informação adicional já citado tem sobre o bem-estar psicológico de pacientes portadoras de câncer de mama. Além da ansiedade, o estudo mais amplo incluirá as variáveis depressão e dor.

\section{MÉTODO}

\section{Amostra}

A investigação foi realizada com de 22 pacientes do Serviço de Mastologia do Hospital de Clínicas de Porto Alegre (HCPA), internadas no período de julho a novembro de 2000 para a realização de mastectomia, com idades variando de 39 a 77 anos, as quais concordaram em participar da pesquisa nas condições especificadas no termo de consentimento informado. Não foram incluídas na amostra pacientes que apresentassem histórico de enfermidade psíquica grave, que já tivessem realizado uma mastectomia anterior ou que fossem analfabetas.

\section{Instrumentos}

\section{Ficha de identificação}

Utilizou-se uma ficha de identificação que coletou informações como nome completo, estado civil, idade, escolaridade, ocupação, renda familiar e número de filhos. Dados provenientes dos prontuários foram utilizados como fonte de informação sobre as condições de saúde e o histórico médico das pacientes, tendo-se em vista os critérios de exclusão adotados.

\section{Inventário de Ansiedade Traço e Estado (IDATE)}

É composto de duas subescalas auto-aplicáveis com 20 itens cada, utilizadas para medir os conceitos de traço e estado de ansiedade. O estado de ansiedade refere-se a um estado emocional transitório, caracterizado por sentimentos de tensão conscientemente percebidos, e é mensurado pelo instrumento através de uma subescala composta de 20 afirmações que devem ser avaliadas pelos sujeitos, segundo a maneira como se sentem em determinado momento. O traço de ansiedade refere-se a diferenças individuais, relativamente estáveis, no modo de o indivíduo reagir a situações ansiogênicas, sendo medido pelo instrumento através dos 20 itens restantes que compõem a segunda subescala, contendo afirmações que requerem dos sujeitos a descrição de como se sentem de um modo geral.Utilizou-se a versão da State-Trait Anxiety Inventory (STAI) de Spielberger, Gorush e Lushene (1970), adaptada para o português e validada por Biaggio e Natalício (1979).

\section{Procedimento de coleta dos dados}

As 22 pacientes pesquisadas foram divididas em dois grupos: observados os critérios de exclusão, as primeiras 11 pacientes internadas durante o período da coleta de dados compuseram o grupo experimental e as 11 pacientes seguintes, o grupo de controle. Todos os sujeitos da amostra, após assinarem o Termo de Consentimento Pós-Informado, completaram a ficha de dados de identificação e os inventários de Ansiedade (IDATE). Somente ao grupo experimental foi apresentado o Vídeo de Informação Adicional. Esses procedimentos, correspondentes ao delineamento experimental clássico antes-depois com dois grupos (Contandriopoulos, Champagne, Potuin, Denis e Boyle, 1997), podem ser melhor visualizados abaixo (Tabela 1):

Quadro 1. Delineamento da pesquisa

\begin{tabular}{|c|c|c|c|}
\hline & Pré-Mastectomia & Intervenção & Pós-Mastectomia \\
\hline \multirow{4}{*}{ 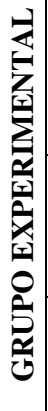 } & $\begin{array}{c}\text { IDATE } \\
\text { (Traço e Estado) }\end{array}$ & \multirow{4}{*}{$\begin{array}{c}\text { Vídeo de } \\
\text { Informação } \\
\text { Adicional }\end{array}$} & $\begin{array}{l}\text { EBP (bem-estar } \\
\text { psicológico) }\end{array}$ \\
\hline & $\begin{array}{l}\text { Questionário de } \\
\text { McGill (treino) }\end{array}$ & & $\begin{array}{c}\text { Questionário de } \\
\text { McGill e Escala } \\
\text { Análogo Visual de Dor }\end{array}$ \\
\hline & \multirow{2}{*}{ BDI } & & IDATE (Estado) \\
\hline & & & Questionário Suporte \\
\hline \multirow{3}{*}{ 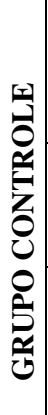 } & $\begin{array}{c}\text { IDATE } \\
\text { (Traço e Estado) }\end{array}$ & \multirow{3}{*}{$\begin{array}{c}\text { Vídeo } \\
\text { ausente }\end{array}$} & $\begin{array}{c}\text { EBP (bem-estar } \\
\text { psicológico) } \\
\text { Questionário de McGill } \\
\text { e Escala Análogo } \\
\text { Visual de Dor } \\
\end{array}$ \\
\hline & $\begin{array}{l}\text { Questionário de } \\
\text { McGill (treino) }\end{array}$ & & IDATE (Estado) \\
\hline & BDI & & Questionário Suporte \\
\hline
\end{tabular}

Vídeo de Informação Adicional ( A preparação do instrumento)

Trata-se de vídeo em formato VHS, concebido, filmado e dirigido pelo próprio pesquisador, com duração aproximada de 30 minutos, contendo informações que procuram reconstituir a rotina da semana de internação da paciente no HCPA para a realização da mastectomia, além de outras informações importantes sobre os procedimentos e cuidados a serem tomados no pós-operatório. 
$\mathrm{O}$ vídeo foi estruturado a partir de informações verbais de profissionais de saúde que atuam diretamente no tratamento e prevenção ao câncer de mama, entre eles enfermeiras e médicos anestesistas, mastologistas e cirurgiões (Vídeo, 2000).

O critério principal para a estruturação do vídeo foi o de compilar imagens e depoimentos que pudessem trazer auxílio para que as pacientes se situassem melhor em relação aos acontecimentos e às especificidades subjacentes à internação. Portanto, as imagens do espaço físico do hospital corresponderiam o máximo possível às reais dependências que seriam utilizadas pelas pacientes ou pelas quais as mesmas transitariam, ao passo que os depoimentos deveriam ser aqueles cujo conteúdo apresentasse informações habitualmente não concedidas nos procedimentos usuais.

Cada profissional que participou do vídeo cirurgião, enfermeira, anestesista - foi convidado a falar sobre vários tópicos propostos pelo pesquisador, tendo por local da entrevista o próprio ambiente hospitalar, estando todos eles vestidos com seus respectivos uniformes de trabalho. A entrevista com a chefe do serviço de voluntárias, bem como os depoimentos de duas ex-pacientes, foram filmados na sala do grupo de apoio durante a reunião semanal do referido grupo. Este procedimento permitiu que o vídeo se aproximasse ainda mais da atmosfera inerente ao hospital e aos procedimentos que nele tomam lugar.

O próximo passo foi a elaboração do roteiro do vídeo a partir do material selecionado. O primeiro formato do vídeo consistia de vinte cenas que compunham um total de oito partes e sete narrações. Esse primeiro formato, após edição realizada em processo digital através de software apropriado, resultou em aproximadamente 40 minutos de duração. A segunda edição do material excluiu cinco narrações e algumas falas consideradas repetitivas ou pouco elucidantes, resultando no formato final do vídeo com vinte cenas divididas em oito partes e cerca de 30 minutos de duração.

\section{RESULTADOS}

\section{Caracterização das participantes}

As 22 pacientes participantes foram estudadas segundo seus dados demográficos obtidos através da ficha de identificação. As freqüências, porcentagens, médias e desvios-padrão, bem como a comparação dos dados demográficos entre os grupos experimental e controle encontram-se na Tabela 2.
Quadro 2. Comparação entre os grupos, freqüências, porcentagens, médias e desvios-padrão dos dados demográficos.

\begin{tabular}{|c|c|c|c|c|c|c|c|}
\hline \multirow{2}{*}{\multicolumn{2}{|c|}{$\begin{array}{c}\text { Dados } \\
\text { Demográficos }\end{array}$}} & \multicolumn{2}{|c|}{$\begin{array}{c}\text { Grupo } \\
\text { Experimental } \\
(n=11)\end{array}$} & \multicolumn{2}{|c|}{$\begin{array}{c}\text { Grupo } \\
\text { Controle } \\
(n=11)\end{array}$} & \multirow[b]{2}{*}{$\mathbf{Z}$} & \multirow[b]{2}{*}{$p$} \\
\hline & & $f$ & $\%$ & $f$ & $\%$ & & \\
\hline \multirow{4}{*}{ 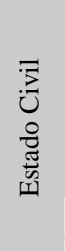 } & casada & 4 & 36,4 & 6 & 54,5 & \multirow{4}{*}{$-0,662$} & \multirow{4}{*}{0,508} \\
\hline & solteira & 3 & 27,3 & 2 & 18,2 & & \\
\hline & viúva & 2 & 18,2 & 1 & 9,1 & & \\
\hline & divorciada & 2 & 18,2 & 2 & 18,2 & & \\
\hline \multirow{5}{*}{ 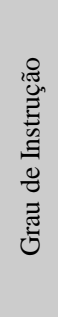 } & alfabetizada & 1 & 9,1 & 1 & 9,1 & \multirow{5}{*}{$-0,170$} & \multirow{5}{*}{0,865} \\
\hline & $1^{\mathrm{o} .}$ grau inc. & 3 & 27,3 & 4 & 36,4 & & \\
\hline & $1^{\circ}$ grau & 2 & 18,2 & 4 & 36,4 & & \\
\hline & $2^{\circ} \cdot$ grau & 4 & 36,4 & 1 & 9,1 & & \\
\hline & superior inc. & 1 & 9,1 & 1 & 9,1 & & \\
\hline \multirow{3}{*}{ 胥: } & classe baixa & 5 & 45,5 & 6 & 54,5 & \multirow{3}{*}{$-0,186$} & \multirow{3}{*}{0,853} \\
\hline & média baixa & 6 & 54,5 & 4 & 36,4 & & \\
\hline & média alta & 0 & - & 1 & 9,1 & & \\
\hline \multirow[t]{2}{*}{ 므 뭉 } & $\mathbf{x}$ & & $\mathbf{s}$ & $x$ & $\mathbf{S}$ & $t$ & $p$ \\
\hline & 49,27 & & 9,84 & 53,19 & 13,46 & 1,587 & 0,456 \\
\hline
\end{tabular}

\section{Resultados da medida de ansiedade}

O coeficiente alfa de Cronbach das escalas de ansiedade aplicadas nas participantes desta pesquisa foi de 0,83 para a de Ansiedade Traço (AT); 0,80 e 0,84 para a de Ansiedade Estado (AE), antes e depois da cirurgia, respectivamente. Estes resultados demonstram uma boa confiabilidade do instrumento para as características desta pesquisa. Não houve diferenças significativas na comparação pré-operatória das pontuações de ansiedade entre os grupos (AE: $p=0,599$; AT: $p=0,974$ ). No entanto, o grupo-controle mostrou variação de AE significativa na comparação deste escore antes e depois da cirurgia $(p=0,050)$. $\mathrm{O}$ estado de ansiedade das pacientes do grupo experimental não teve variação significativa $(p=0,721)$.

\section{DISCUSSÃO DOS RESULTADOS}

As características da amostra em ambos os grupos revelaram homogeneidade no que diz respeito aos dados demográficos pesquisados: idade, renda 
familiar, estado civil e grau de instrução foram equivalentes na comparação entre os dois grupos. $\mathrm{O}$ perfil mais frequiente das pacientes da amostra pode ser definido genericamente como o de uma mulher de aproximadamente 51 anos, casada, com uma prole de dois a três filhos, do lar, cuja formação atinge o primeiro grau completo ou ainda por completar, pertencente à classe de renda baixa ou renda média baixa (Tabela 1). Os resultados dos testes préoperatóros referentes à variável de controle Ansiedade (Traço e Estado) -, também revelaram homogeneidade entre os grupos. A soma desses resultados pré-operatórios garantiu um bom controle das variáveis intervenientes.

Os níveis de estado de ansiedade mostraram-se mais elevados no grupo controle, de acordo com as variações significativas detectadas na comparação pré e pós-operatória nesse grupo.

O número expressivo de estudos que citam a influência da ansiedade (Payne e cols., 1999; Thomas e cols., 1998; Farragher, 1998; Morasso, 1997; O’Mahoney e Carrol, 1997; Wallis e cols., 1997; Becker e cols., 1997; Kuecuekdeveci e cols., 1996; Tasmuth e cols., 1996; Page e cols., 1995), no que tange ao bem-estar e à qualidade de vida dos pacientes, leva a crer que se possa admitir que o grupo experimental tenha apresentado melhores condições em termos de bem-estar, no que se refere à ansiedade, durante os dias de internação. É importante salientar que esses resultados mais positivos no estado de ansiedade obtidos pelo grupo experimental já se mostraram estatisticamente significativos, não obstante o reduzido tamanho da amostra. Dessa forma, parece aconselhável o aumento do tamanho da amostra para que mais luz seja lançada sobre a questão e que se possa chegar a conclusões mais sólidas. Observa-se, ainda, que, nessas condições se faz necessário que os resultados sejam entendidos com alguma restrição e que todas as conclusões tiradas a partir deles sejam consideradas válidas apenas para a amostra pesquisada. No entanto, reitera-se que as significâncias estatísticas atingidas, mesmo com o número reduzido de casos, indicam a validade $\mathrm{e}$ pertinência do processo de aumento da amostra para que as conclusões se tornem mais confiáveis.

A preocupação na área da saúde tem sido direcionada nos últimos tempos nem tanto à cura de doenças, mas à sua profilaxia. Isso não se dá apenas devido a fatores de bem-estar social, mas também àqueles de ordem econômica. Uma população com altos íjdices de adoecimento pode causar grandes prejuízos aos cofres públicos, situação que se agrava em países com menos recursos alocados à saúde, como o Brasil. Mas a busca por melhores condições de atendimento das demandas de saúde da população supõe avanços no conhecimento sobre os aspectos inerentes ao problema. Esses avanços não se restringem à tecnologia ou à bioquímica, mas se fazem necessários igualmente na compreensão e acolhimento de aspectos emocionais e subjetivos do adoecer.

A ansiedade, além da sua considerável ocorrência nas pacientes após o diagnóstico de câncer de mama e principalmente após a mastectomia, representa um fator de muita influência no sentimento de bem-estar das pessoas de um modo geral (Payne e cols., 1999, Baseggio, 1996, Medeiros e Scherman,2001). Sob esse aspecto, os primeiros resultados desta pesquisa são animadores, ao passo que indicam que o procedimento de informação pode se tornar elemento valioso na melhora da condição de aspectos que influem diretamente no bem-estar das pacientes mastectomizadas.

A necessidade do atendimento multidisciplinar às demandas de informações das pacientes pode trazer problemas de ordem prática. Parece ser impossível, no contexto do serviço hospitalar, organizar um procedimento completo de informações a partir da mobilização simultânea de todo $\mathrm{o}$ grupo de profissionais responsáveis pelo cuidado das pacientes. As informações obtidas através das consultas prévias à cirurgia e as recebidas durante a internação acabam constituindo um material fragmentado e, portanto, de difícil compreensão global por parte das pacientes.

Entre as soluções possíveis para esse problema está a utilização de recursos audiovisuais. Um dos recursos bastante acessíveis modernamente é o vídeo. Estudos comprovam que cerca de $95 \%$ da aprendizagem do ser humano se dá por meio do ouvido e da visão associados, assim como conteúdos audiovisuais tendem a ser mais facilmente memorizados e retidos do que se fossem apenas vistos ou escutados em separado (Ferrés, 1996).

$\mathrm{Na}$ última década, pesquisas que buscam novos procedimentos na área clínica têm obtido bons resultados com a utilização do vídeo como auxiliar em tratamentos psicoterápicos, ressaltando a característica motivadora dessa tecnologia (Maggi, 1999). Nesse sentido, parece ser útil a investigação dos efeitos de um procedimento que utiliza uma tecnologia moderna como a do vídeo, como alternativa de trabalho com pacientes cirúrgicos.

\section{REFERÊNCIAS BIBLIOGRÁFICA}

Appels A., Bar, F., Lasker, J. \& Flamm. U. (1997). The effect of a psychological intervention program on the risk of a new 
coronary event after angioplasty: A feasibility study. Journal of Psychosomatic Research, 43 (2), 209-217.

Baseggio, D. L. (Org.) (1999). Câncer de mama: Abordagens multidisciplinares. Rio de Janeiro: Revinter.

Becker, N., Thomsen, A. B., Olsen, A. K., Sjogren, P., Bech, P. \& Eriksen, J. (1997). Pain epidemiology and health related quality of life in chronic non-malignant pain patients referred to a Danish multidisciplinary pain center. $\underline{\text { Pain }}, \underline{73}$ (3), 393-400.

Biaggio, A.M.B., NATALÍCIO, L. (1979). Inventário de ansiedade traço-estado - IDATE. Manual. Rio de Janeiro, RJ : Psicologia Aplicada.

Breemhaar, B., Van Den Borne, H. W. \& Mullen, P.D. (1996). Inadequacies of surgical and patient education. Patient Education and Counseling, 28 (1), 31-44.

Brum, A. F. M., Chem, R. C., Peres, L.M. \& Magalhães, M. A. (1997). Repercussões psicológicas frente ao manejo cirúrgico do câncer de mama: uma revisão. Revista AMRIGS, 41 (2), 79-83.

Campos, T. C. P. (1995). Psicologia Hospitalar: a atuação do psicólogo em hospitais. São Paulo : EPU.

Clewes, J. L. \& Endler, N. S. (1994). State-trait anxiety and the experience of elective surgery in children. Canadian Journal of behavioural Science, 26 (2), 183-198.

Contandriopoulos, A-P., Champagne, F., Potuin, L., Denis, J-L. \& Boyle, P. (1997). Saber preparar uma pesquisa: definição estrutura e financiamento ( $2^{\mathrm{a}}$ ed.) São Paulo/ Rio de Janeiro: Hucitec/Abrasco.

Farragher, B. (1998). Psychiatric morbidity following the diagnosis and treatment of early breast cancer. Irish Journal of Medical Science, 167, (3) 166-169.

Fernández-Ríos, L. (1994). Manual de Psicologia Preventiva: teoria y práctica. ( $1^{a}$ ed.) s/l.: Siglo Veintiuno de España Editores.

Ferrell, B. R., Grant, M., Funk, B., Garcia, N., Otis Green, S. \& Schaffner M. L. (1996). Quality of life in breast cancer. Cancer Practice, 4 , (6), 331-340.

Ferrés, J. (1996). Vídeo e educação. (2 $2^{\mathrm{a}}$ ed.) Porto Alegre: Artes Médicas.

Glanz, K., \& Lerman, C. (1992). Psychosocial impact of breast cancer: A critical review. Annals of Behavioral Medicine. 14 (3), 204-212.

Heywood, P. L., Blackie, G. C, Cameron, I. H. \& Dowell, A. C. (1998). An assessment of the attributes of frequent attendees to general practice. Family Practice. 15, (3) 198-204.

Horne, D. J. De L, Vatmanidis, P. \& Careri, A. (1994). Preparing patients for invasive medical and surgical procedures: Adding behavioral and cognitive interventions. Behavioral Medicine, 20 (1), 5-13.

Kuecuekdeveci, A. A., Tennant, A., Hardo, P. \& Chamberlain, M. A. (1996). Sleep problems in stroke patients: Relationship with shoulder pain. Clinical Rehabilitation. 10 (2), 166-172.

Levinson, W. \& Chaumeton, N. (1999). Communication between surgeons and patients in routine office visits. Surgery 125 (2).
NATIONAL Cancer Institute. (2000). Estimativas de incidência de neoplasia no Brasil. Disponível em: <http://www.mci.nih.gov/cancerinfo/index.html>. (Acessado em 04/12/2001)

Maggi, A. (1999). Intervencão psicológica com pais de crianças portadoras de fissuras labiais e/ou palatais. Tese de Doutorado, Pontifícia Universidade Católica do Rio Grande do Sul, Porto Alegre.

Medeiros, R. H. A., Scherman, L. (1999). Questionário Suporte. Não publicado.

Medeiros, R. H. A., Scherman, L. (2001). Fatores Intervenientes no Bem-Estar Psicológico de Pacientes Submetidas à Mastectomia. Revista de Medicina da PUCRS. 11 (1), 44-50.

Morasso, G. (1997). Screening adjustment disorders related to mastectomy and its treatment. New Trends in Experimental and Clinical Psychiatry 13 (1), 90-93.

Nuñez, R. N., Sanabria, R. M., Guerrero, F. R. \& Sanchez, E. B. (1991). Metodos de valoracion del dolor postoperatorio en el niño. Cirurgia Pediatrica. 4 (2), 60-65.

O'Mahoney, J. M. \& Carroll, R. A. (1997). The impact of breast cancer and its treatment on marital functioning. Journal of Clinical Psychology in Medical Settings. 4 (4), 397-415.

Page, S. A., Verhoef, M. J. \& Emes, C. G. (1995). Quality of life, bypass surgery and the elderly. Canadian Journal of Cardiology. 11 (9), 777-782.

Payne, D. K., Hoffman, R. G., Theodoulou, M., Dosik, M. \& Massie, M. J. (1999). Screening for anxiety and depression in women with breast cancer: Psychiatry and medical care gear up for managed care. Psychosomatic. $\underline{40}$ (1), 64-69.

Spielberger, C.D., Goruch, R.L., Lushene, R E. (1970). Manual for the STAI. Palo Alto, California: Consulting Psychologist.

Tasmuth, T., Estlanderb, A. M. \& Kalso E. (1996). Effect of present pain and mood on the memory of past postoperative pain in women treated surgically for breast cancer. Pain. 68 ( 2 - 3), 343-347.

Thomas, T., Robinson, C., Champion, D., Mckell, M. \& Pell, M. (1998). Prediction and assessment of the severity of post-operative pain and of satisfaction with management. Pain. 75 (2 - 3), 177-185.

VÍDEO de informação adicional. Roberto H. Amorim de Medeiros. Porto Alegre, 2000. 1 videocassete [30 min] : son., color.; $12 \mathrm{~mm}$ VHS NTSC.

Wallis, B. J., Lord, S. M. \& Bogduk, N. (1997). Resolution of psychological distress of whiplash patients following treatment by radiofrequency neurotomy: A randomised, double-blind, placebocontrolled trial. Pain. 73 (1), 15-22.

Recebido em 19/06/2001

Revisado em 20/11/2001

Aceito em 30/11/2001 